\title{
Metabolic syndrome, insulin resistance and sleepiness in real-life obstructive
} sleep apnoea

\author{
Maria R. Bonsignore*, Cristina Esquinas ${ }^{\#, \text {, Antonia Barceló }}$, \\ Manuel Sanchez-de-la-Torre ${ }^{\S, f}$, Alessandra Paternó*, Joaquin Duran-Cantolla**, \\ José M. Marín ${ }^{\# \#}$ and Ferran Barbé ${ }^{\uparrow, \$}$
}

ABSTRACT: The metabolic syndrome shows a variable prevalence in obstructive sleep apnoea (OSA), and its association with insulin resistance or excessive daytime sleepiness in OSA is unclear. This study assessed the following in consecutive patients with newly diagnosed OSA: 1) the prevalence of metabolic syndrome; and 2) its association with insulin resistance and daytime sleepiness.

Metabolic syndrome (National Cholesterol Education Program Adult Treatment Panel (NCEPATP) III criteria), insulin resistance (Homeostatic Model Assessment (HOMA) index, $n=288$ ) and daytime sleepiness (Epworth Sleepiness Scale) were assessed in 529 OSA patients.

The prevalence of metabolic syndrome was $51.2 \%$, which increased with OSA severity. Each metabolic syndrome component correlated with apnoea/hypopnoea index, but only blood pressure retained significance after correction for confounders. Both obesity and OSA contributed to metabolic abnormalities, with different sex-related patterns, since diagnosis of metabolic syndrome was significantly associated with neck circumference, age, body mass index and lowest arterial oxygen saturation in males, and with age and arousal index in females. The number of metabolic syndrome components increased with HOMA index $(p<0.001)$. Prevalence of sleepiness was the same in patients with and without metabolic syndrome.

The metabolic syndrome occurs in about half of "real-life" OSA patients, irrespective of daytime sleepiness, and is a reliable marker of insulin resistance.

KEYWORDS: Epidemiology, intermittent hypoxia, metabolism, sex

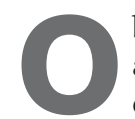

bstructive sleep apnoea (OSA) is often associated with obesity, hypertension and other cardiovascular risk factors [1], and untreated patients with severe OSA show an increased risk for cardiovascular morbidity and mortality $[2,3]$. However, since OSA and obesity frequently coexist, their respective role in increased cardiovascular risk is still debated.

Several studies have shown that insulin resistance occurs in OSA patients and directly correlates with OSA severity (for review see [4]). Besides obesity, OSA may play an independent role in the pathogenesis of insulin resistance, since intermittent hypoxia was shown to cause insulin resistance in healthy humans [5]. However, the available data are somewhat controversial, since the association of OSA and insulin resistance was mostly accounted for by obesity in at least four studies [6-9], and short-term treatment of OSA with continuous positive airway pressure (CPAP) failed to improve metabolic abnormalities [4].

The metabolic syndrome is a cluster of risk factors associated with insulin resistance, increased risk for type 2 diabetes [10] and increased overall and cardiovascular mortality [11]. Although its value in cardiovascular risk prediction is debated, the concept of metabolic syndrome has gained popularity and improved clinicians' awareness of metabolic problems in obese subjects [12]. According to the latest National Cholesterol Education Program Adult Treatment Panel (NCEP-ATP) III definition [13], the metabolic syndrome is diagnosed when at least three of the following conditions occur: increased waist circumference, as a marker of central obesity; increased blood pressure; fasting hyperglycaemia; increased serum triglyceride and decreased high-density lipoprotein (HDL) cholesterol concentrations.

\section{AFFILIATIONS}

*Biomedical Dept of Internal and Specialist Medicine (DIBIMIS), Section of Pneumology, University of Palermo, Italy.

${ }^{\#}$ Sleep Apnoea Unit, Hospital Santa Maria,

"Hospital Universitario Arnau de Vilanova, Respiratory Dept,

${ }^{\S}$ Respiratory Diseases Research Unit, IRB Lleida, Lleida,

${ }^{+}$Clinical Analysis Service, Hospital Universitari Son Dureta, Palma de Mallorca,

${ }^{f}$ Centro de Investigación Biomédica en Red de Enfermedades

Respiratorias (CIBERES), Instituto

Nacional de Salud Carlos III, Madrid,

**Sleep Disorders Unit, Universitary Hospital of Alava, University of the

Basque Country, Vitoria, and

\#\#Pneumology Dept, Hospital

Miguel Servet, Zaragoza, Spain.

CORRESPONDENCE

M.R. Bonsignore

Biomedical Dept of Internal and

Specialist Medicine (DIBIMIS),

Section of Pneumology

University of Palermo

Via Trabucco 180

Palermo

Italy

E-mail:marisa@ibim.cnr.it

Received:

Sept 272010

Accepted after revision:

Sept 192011

First published online:

Nov 102011 
Prevalence of the metabolic syndrome in OSA patients according to the NCEP-ATP III definition was found to range between $23 \%$ and $87 \%$ [14-19]. Most studies included small numbers of patients and did not assess insulin resistance in conjunction with metabolic syndrome. One case-control study [7] and a cross-sectional population study [20] suggested that metabolic syndrome, but not insulin resistance, was associated with OSA. Surprisingly, the value of metabolic syndrome in predicting insulin resistance has not been specifically tested in OSA patients. Therefore, our first aim was to assess the prevalence of the metabolic syndrome in a large sample of consecutive OSA patients at diagnosis and to compute relationships between sleep characteristics, insulin resistance and metabolic abnormalities.

Excessive daytime sleepiness (EDS) is a major symptom of OSA. EDS in OSA patients was reported to be associated with hypertension [21], altered autonomic modulation [22] and type 2 diabetes [23]. EDS was among the factors significantly associated with OSA and metabolic syndrome in the recent study by AgraWAL et al. [18]. Two case-control studies have found that EDS predicted insulin resistance in OSA patients independently of obesity [24, 25]; only sleepy patients showed improved insulin sensitivity after CPAP treatment for 3 months [24]. Conversely, other studies have found a similar degree of subjective sleepiness in metabolic syndrome patients with or without OSA [26]. Therefore, the second aim of the current investigation was to assess the characteristics of OSA patients reporting daytime sleepiness and whether EDS is associated with metabolic syndrome in a large series of OSA patients.

\section{METHODS \\ Patients}

Consecutive patients referred to the Sleep Laboratory, Hospital Son Dureta, Palma de Mallorca, Spain, in the years 2005-2007 were studied $(n=535)$. The inclusion criteria were age $>18 \mathrm{yrs}$, diagnosis of OSA and wish to participate to the study. No eligible patient refused to participate. Six patients were excluded due to missing data, reducing the sample to 529 patients. The study protocol was approved by the local Institutional Review Board (approval number IB741/09PI), and all participants gave their informed written consent.

\section{Sleep study}

OSA was diagnosed by full polysomnography (E-Series Compumedics, Abbotsford, Australia) that included recording of oronasal flow, thoraco-abdominal movements, ECG, submental and pretibial electromyography, electrooculogram, electroencephalogram and pulse oximetry, as previously described [22]. Apnoea was defined by absence of airflow lasting $\geqslant 10 \mathrm{~s}$. Hypopnoea was defined as any airflow reduction lasting $\geqslant 10 \mathrm{~s}$ associated with either oxygen desaturation $\geqslant 4 \%$ or arousal and oxygen desaturation $\geqslant 3 \%$. The apnoea/hypopnoea index (AHI) and the arousal index (ArI) were defined as the number of apnoeas and hypopnoeas, and of arousals, respectively, per hour of sleep. OSA was diagnosed if AHI was $\geqslant 10$ events $\cdot h^{-1}$. EDS, quantified by the Epworth Sleepiness Scale (ESS), was defined as an ESS score $\geqslant 10$.

\section{Metabolic syndrome}

As a general measure of obesity, body mass index (BMI) was defined as weight divided by height ${ }^{2}$ (in $\mathrm{kg} \cdot \mathrm{m}^{-2}$ ). Neck and waist circumferences (in $\mathrm{cm}$ ) were also measured. The metabolic syndrome was diagnosed based on the presence of three or more of the following factors: waist circumference $\geqslant 80 \mathrm{~cm}$ in females and $\geqslant 94 \mathrm{~cm}$ in males (all patients were Caucasian); serum triglycerides $\geqslant 150 \mathrm{mg} \cdot \mathrm{dL}^{-1}$ or lipid-lowering treatment; HDL cholesterol $<40 \mathrm{mg} \cdot \mathrm{dL}^{-1}$ in males, $<50 \mathrm{mg} \cdot \mathrm{dL}^{-1}$ in females, or lipid-lowering treatment; increased blood pressure or anti-hypertensive treatment; and fasting blood glucose $>100 \mathrm{mg} \cdot \mathrm{dL}^{-1}$ or anti-diabetic treatment [13].

Office blood pressure was measured by a standard mercury sphygmomanometer while the subject was quietly seated after $\geqslant 5 \mathrm{~min}$ of rest. Increased blood pressure was recorded if systolic blood pressure was $>130 \mathrm{mmHg}$ or diastolic pressure was $>85 \mathrm{mmHg}$, or the patient was on anti-hypertensive treatment.

Fasting venous blood samples were obtained in the morning after polysomnography. Glucose, triglycerides, total cholesterol and HDL cholesterol were determined by standard enzymatic methods on a Hitachi Modular analyser (Roche Diagnostics, Indianapolis, IN, USA). In 288 patients without previously known diabetes, plasma insulin concentration was measured by chemiluminescent assays on an Immulite 2000 analyser (Siemens Medical Solutions Diagnostics, New York, NY, USA). Insulin resistance was calculated using the Homeostatic Model Assessment (HOMA) index [27].

\section{Statistical analysis}

Data are presented as mean $\pm \mathrm{SD}$; categorical data are shown as percentage of positive patients.

Unpaired t-tests (for numerical variables) or nonparametric Mann--Whitney U-tests (for variables that were not normally distributed) were used to compare: 1) patient characteristics according to sex; 2) patients without any metabolic syndrome components versus all other patients; 3) patients with and without a diagnosis of metabolic syndrome; and 4) patients with and without EDS. Frequencies were compared by the Chisquared test for categorical variables (Fisher's exact test with observed frequencies $<5$ ). Due to non-normal distribution, HOMA index and serum triglyceride values were analysed after logarithmic transformation.

Trends were analysed by the Spearman rank test, or the Kendall Tau-c test for categorical variables. Multiple linear regression was used to assess the relationship between $\mathrm{AHI}$, ArI and arterial oxygen saturation $\left(\mathrm{Sa}_{2} \mathrm{O}_{2}\right)$ as independent variables and each metabolic syndrome component as dependent variable.

The multivariate logistic regression model was used to assess determinants of metabolic syndrome and EDS. To this aim, we used anthropometric and sleep variables together with the variables showing $\mathrm{p}<0.20$ in bivariate analysis. Variables were selected using a stepwise approach. A p-value $<0.05$ was considered significant. The SPSS version 17 software (SPSS Inc., Chicago, IL, USA) was used for all analyses.

\section{RESULTS}

\section{Metabolic syndrome}

Total sample and sex-specific characteristics are reported in table 1. OSA patients were mostly male and obese. Females accounted for about one-fifth of the total sample $(n=105)$. Neck 


\begin{tabular}{|c|c|c|c|}
\hline $\begin{array}{l}\text { Anthropometric } \\
\text { sample }\end{array}$ & and sleep c & haracteris & ics of the \\
\hline & All patients & Males & Females \\
\hline Subjects $n$ & 529 & 424 & 105 \\
\hline Age yrs & $51.3 \pm 12.8$ & $50.9 \pm 13.0$ & $52.9 \pm 12.0$ \\
\hline Hypertension & 35.2 & 36.6 & 29.5 \\
\hline Dyslipidaemia & 32.5 & 35 & 30.0 \\
\hline Diabetes mellitus & 17.0 & 16.5 & 19 \\
\hline BMI $\mathbf{k g} \cdot \mathrm{m}^{-2}$ & $30.8 \pm 6.0$ & $30.6 \pm 5.4$ & $31.6 \pm 7.9$ \\
\hline Obesity & 49 & 48.3 & 51.4 \\
\hline Neck circumference $\mathrm{cm}$ & $41.6 \pm 4.1$ & $42.7 \pm 3.5$ & $37.3 \pm 3.8^{* * *}$ \\
\hline AHI events $\cdot h^{-1}$ & $43.4 \pm 27.6$ & $44.8 \pm 26.7$ & $37.7 \pm 30.7^{+}$ \\
\hline Arl events $\cdot h^{-1}$ & $51.5 \pm 23.2$ & $52.4 \pm 22.6$ & $47.8 \pm 25.3$ \\
\hline Mean nocturnal $\mathrm{Sa}, \mathrm{O}_{2} \%$ & $92.3 \pm 4.0$ & $92.1 \pm 4.0$ & $93.0 \pm 4.0$ \\
\hline Lowest $\mathrm{Sa}, \mathrm{O}_{2} \%$ & $80.8 \pm 9.3$ & $80.5 \pm 9.3$ & $81.6 \pm 9.4$ \\
\hline ESS score & $9.7 \pm 5.1$ & $9.8 \pm 5.0$ & $9.5 \pm 5.5$ \\
\hline Daytime sleepiness ${ }^{\pi}$ & 51.8 & 51.4 & 53.3 \\
\hline Diagnosis of metabolic syndrome & 51.2 & 51.4 & 50.5 \\
\hline High blood pressure & 54.6 & 55.4 & 51.4 \\
\hline High waist circumference & 72 & 70.8 & 77.1 \\
\hline Low HDL cholesterol & 26.8 & 26.7 & 27.6 \\
\hline High triglyceride level & 45.9 & 47.6 & 39 \\
\hline High fasting blood glucose & 49.9 & 50.7 & 46.7 \\
\hline
\end{tabular}

Data are presented as mean \pm SD or $\%$, unless otherwise stated. BMI: body mass index; $\mathrm{AHI}$ : apnoea/hypopnoea index; Arl: arousal index; $\mathrm{Sa}_{1} \mathrm{O}_{2}$ : arterial oxygen saturation; ESS: Epworth Sleepiness Scale; HDL: high-density lipoprotein. ${ }^{*}:$ : BMl $\geqslant 30 \mathrm{~kg} \cdot \mathrm{m}^{-2}$; 9 : ESS $\geqslant 10$. ${ }^{* *}: \mathrm{p}<0.001 ;{ }^{+}: \mathrm{p}=0.02$ for difference between sexes by unpaired t-test or Chi-squared analysis. circumference and AHI were significantly higher in males than in females. Current smokers accounted for $29 \%$ of the subjects, while chronic obstructive pulmonary disease or cardiovascular disease (coronary artery disease and heart failure) occurred in $6.6 \%$ and $5.9 \%$ of the subjects, respectively, without differences between sexes (data not shown).

The overall prevalence of the metabolic syndrome was $51.2 \%$. The number of metabolic syndrome components increased with OSA severity (table 2 and fig. 1). Mild, moderate and severe OSA patients showed significantly different distributions of metabolic syndrome components, with mild patients often being free of any metabolic syndrome component, and severe patients frequently showing three to five metabolic syndrome components. Patients with one or two metabolic syndrome components were classified with all degrees of OSA severity without any specific distribution pattern (fig. 1).

The distribution and prevalence of metabolic syndrome components in the sample are shown in figure 2 . The most common combinations of metabolic syndrome components included increased waist circumference, hypertension and abnormal fasting blood glucose (table S1).

Some patients $(n=55)$ showed no metabolic syndrome components. Compared with patients with one or more metabolic syndrome components, they were non-obese, had mild to moderate OSA, were significantly younger (table S2), and included more active smokers (data not shown).

Markers of OSA severity (AHI and mean $\mathrm{Sa}_{2} \mathrm{O}_{2}$ ) (table 3 ) showed significant unadjusted linear relationships with each component of the metabolic syndrome. Most of the significance was lost for AHI after adjustments for age, BMI, smoking and

\begin{tabular}{|c|c|c|c|c|c|c|c|}
\hline & \multicolumn{6}{|c|}{ Number of metabolic syndrome components } & \multirow[t]{2}{*}{ Rho/tau } \\
\hline & 0 & 1 & 2 & 3 & 4 & 5 & \\
\hline Subjects $n$ & 55 & 81 & 121 & 134 & 94 & 44 & \\
\hline Age yrs & $40.9 \pm 13.1$ & $46.6 \pm 12.0$ & $52.5 \pm 12.5$ & $52.9 \pm 11.8$ & $54.3 \pm 11.5$ & $55.9 \pm 13.4$ & $0.305^{\star \star \star}$ \\
\hline Diabetes mellitus & 0 & 0 & 10 & 20.5 & 36.4 & 47.7 & $0.338^{* \star *}$ \\
\hline BMI $\mathbf{k g} \cdot \mathrm{m}^{-2}$ & $24.5 \pm 2.7$ & $28.2 \pm 4.6$ & $29.7 \pm 5.2$ & $32.5 \pm 5.5$ & $34.2 \pm 6.1$ & $34.4 \pm 5.6$ & $0.548^{\star \star \star}$ \\
\hline Obesity & 3.7 & 28.0 & 48.6 & 68.6 & 81 & 84.6 & $0.517^{\star \star *}$ \\
\hline Neck circumference $\mathrm{cm}$ & $37.5 \pm 3.2$ & $39.6 \pm 3.7$ & $41.1 \pm 3.3$ & $42.3 \pm 3.7$ & $44.2 \pm 4.1$ & $43.8 \pm 4.0$ & $0.462^{\star * *}$ \\
\hline AHI events $\cdot h^{-1}$ & $22.9 \pm 17.6$ & $31.5 \pm 20.8$ & $36.8 \pm 23.5$ & $49.9 \pm 26.8$ & $56.2 \pm 28.1$ & $61.9 \pm 31.2$ & $0.428^{* \star \star}$ \\
\hline Arl events $\cdot h^{-1}$ & $34.3 \pm 16.9$ & $41.6 \pm 17.0$ & $46.1 \pm 20.7$ & $56.2 \pm 22.6$ & $64.2 \pm 23.5$ & $65.0 \pm 22.4$ & $0.428^{* * *}$ \\
\hline Mean nocturnal $\mathrm{Sa}, \mathrm{O}_{2} \%$ & $95.3 \pm 2.0$ & $94.1 \pm 2.3$ & $93.2 \pm 2.8$ & $91.5 \pm 4.1$ & $90.2 \pm 4.6$ & $90.2 \pm 4.9$ & $-0.466^{\star \star \star}$ \\
\hline Lowest $\mathrm{Sa}, \mathrm{O}_{2} \%$ & $87.1 \pm 5.7$ & $84.6 \pm 6.7$ & $83.1 \pm 7.2$ & $78.2 \pm 9.4$ & $77.6 \pm 9.8$ & $74.3 \pm 11.5$ & $-0.419 * \star \star$ \\
\hline ESS score & $9.9 \pm 5.2$ & $9.7 \pm 5.3$ & $9.0 \pm 4.9$ & $9.7 \pm 5.1$ & $10.5 \pm 4.9$ & $9.7 \pm 5.6$ & 0.036 \\
\hline Systolic blood pressure $\mathrm{mmHg}$ & $113.3 \pm 8.5$ & $121.7 \pm 13.7$ & $130.3 \pm 16.9$ & $135.0 \pm 15.2$ & $139.3 \pm 17.7$ & $144.1 \pm 10.5$ & $0.522^{\star \star *}$ \\
\hline Diastolic blood pressure $\mathrm{mmHg}$ & $68.0 \pm 9.0$ & $74.6 \pm 9.4$ & $79.6 \pm 11.5$ & $82.6 \pm 12.3$ & $85.2 \pm 11.2$ & $86.8 \pm 10.7$ & $0.429 * \star \star$ \\
\hline Waist circumference $\mathrm{cm}$ & $88.9 \pm 9.3$ & $99.8 \pm 11.5$ & $105.3 \pm 11.1$ & $112.4 \pm 12.0$ & $115.4 \pm 13.1$ & $114.8 \pm 11.5$ & $0.575^{\star \star \star}$ \\
\hline $\mathrm{HDL}$ cholesterol $\mathrm{mg} \cdot \mathrm{dL}^{-1}$ & $60.1 \pm 13.6$ & $57.1 \pm 14.9$ & $57.4 \pm 22.7$ & $51.3 \pm 10.6$ & $49.8 \pm 22.3$ & $41.6 \pm 11.7$ & $-0.363^{\star \star *}$ \\
\hline Triglycerides $\mathbf{m g} \cdot \mathrm{dL}^{-1}$ & $85.6 \pm 31.4$ & $113.3 \pm 58.0$ & $129.8 \pm 54.2$ & $160.0 \pm 74.3$ & $205.5 \pm 94.8$ & $245.8 \pm 132.1$ & $0.598^{\star \star *}$ \\
\hline Fasting blood glucose $\mathrm{mg} \cdot \mathrm{dL}^{-1}$ & $88.5 \pm 6.6$ & $91.8 \pm 6.5$ & $101.8 \pm 26.1$ & $110.1 \pm 24.7$ & $117.7 \pm 27.5$ & $128.7 \pm 30.0$ & $0.614^{\star \star \star}$ \\
\hline
\end{tabular}

Data are presented as mean \pm SD or \%, unless otherwise stated. BMI: body mass index; AHI: apnoea/hypopnoea index; Arl: arousal index; Sa, $\mathrm{O}_{2}$ : arterial oxygen saturation; ESS: Epworth Sleepiness Scale; HDL: high-density lipoprotein. ${ }^{*}$ : BMI $\geqslant 30 \mathrm{~kg} \cdot \mathrm{m}^{-2}$. ${ }^{\star *}$ : $\mathrm{p}<0.001$ for trend. 


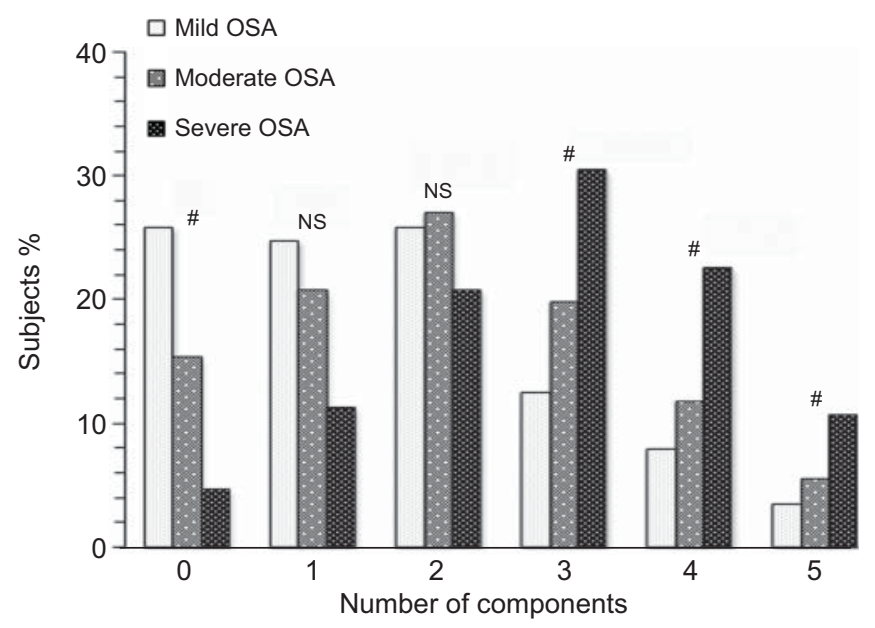

FIGURE 1. Obstructive sleep apnoea (OSA) severity and number of metabolic syndrome components. Patients with mild OSA (apnoea/hypopnoea index (AHI) $<15$ events $\cdot h^{-1}$ ) were often free of any metabolic syndrome component. Conversely, patients with severe OSA (AHI $>30$ events. $h^{-1}$ ) had a metabolic syndrome diagnosis (i.e. three or more components) more frequently than the other two groups. Distribution of mild, moderate and severe OSA did not differ in patients with one or two metabolic syndrome components. ${ }^{\#}: p<0.0001$ by Chi-squared test; NS: nonsignificant.

sex, except for systolic and diastolic blood pressure. Mean $\mathrm{Sa}_{1} \mathrm{O}_{2}$ remained significantly associated with waist circumference and diastolic blood pressure after adjustments. ArI and lowest $\mathrm{Sa}_{1} \mathrm{O}_{2}$ were also analysed, with results similar to those obtained for $\mathrm{AHI}$ and mean $\mathrm{Sa}_{2} \mathrm{O}_{2}$, respectively (table $\mathrm{S} 3$ ).

\section{Insulin resistance}

Insulin resistance, estimated by the HOMA index in 288 patients, increased with increasing number of metabolic syndrome components (Spearman's rho 0.455; p<0.001) (fig. 3 and table 4). The HOMA index correlated positively with AHI and ArI, and negatively with lowest or mean $\mathrm{Sa}_{1} \mathrm{O}_{2}$ in unadjusted bivariate analysis $(\mathrm{p}<0.0001)$ (data not shown). All such relationships

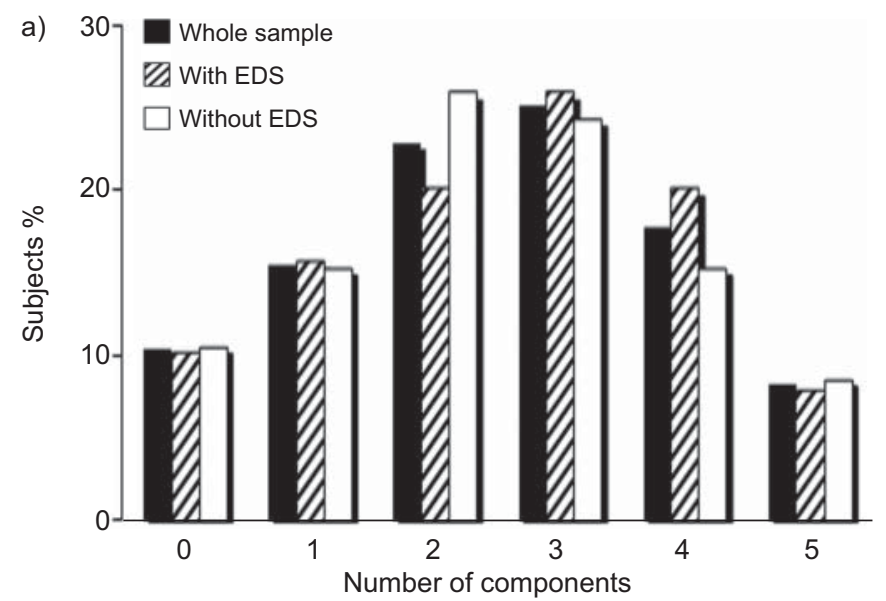

became nonsignificant after adjustment for BMI and were unaffected by sex (data not shown).

\section{Sleepiness}

The prevalence of the metabolic syndrome, or of each of its components, was similar in patients with and without EDS (fig. 2). The relationship between HOMA index and number of metabolic syndrome components was comparable in OSA patients with $(0.466 ; \mathrm{p}<0.001)$ and without EDS $(0.426$; p <0.001) (fig. 3). ESS and HOMA index did not show any significant relationship. Patients free from any metabolic syndrome component showed a similar EDS to the rest of the sample (table S2). No difference was found in EDS between patients with and without a metabolic syndrome diagnosis (table 5). Patients with EDS were younger, showed a slightly higher waist circumference and worse polysomnographic variables, but similar blood pressure and other metabolic variables compared with nonsleepy patients (table 5).

\section{Logistic regression analysis}

To assess the factors associated with the metabolic syndrome in the whole sample, the following variables were entered into the model: EDS, BMI, neck circumference, sex, age, AHI, ArI, apnoea index, lowest and mean nocturnal $\mathrm{Sa}_{2} \mathrm{O}_{2}$. The metabolic syndrome in OSA was significantly associated with the following: sex (OR 1.033, 95\% CI 1.013-1.054; $p=0.001$ ); neck circumference (OR 1.174, 95\% CI 1.057-1.304; $\mathrm{p}=0.003$ ); ArI (OR 1.027, 95\% CI 1.005-1.048; $\mathrm{p}=0.014$ ); BMI (OR 1.083, 95\% CI 1.010-1.162; $\mathrm{p}=0.03)$; lowest nocturnal $\mathrm{Sa}_{2} \mathrm{O}_{2}(\mathrm{OR} 0.953,95 \%$ CI $0.910-0.997 ; p=0.036)$. Age showed a strong trend (OR 1.033, 95\% CI 0.998-5.3; $\mathrm{p}=0.05)$, while EDS did not contribute significantly (OR 1.220, 95\% CI 0.780-1.907; p=nonsignificant).

To better explore sex-related differences, the analysis was repeated separately in males and females. In males, the metabolic syndrome was significantly associated with neck circumference, age, BMI and lowest nocturnal $\mathrm{Sa}_{2} \mathrm{O}_{2}$, with the regression accounting for $42.3 \%$ of the variability. In females, ArI and age explained $52.4 \%$ of metabolic syndrome variability, and lowest nocturnal $\mathrm{Sa}_{2} \mathrm{O}_{2}$ showed a strong trend for association with metabolic syndrome $(p=0.053)$.

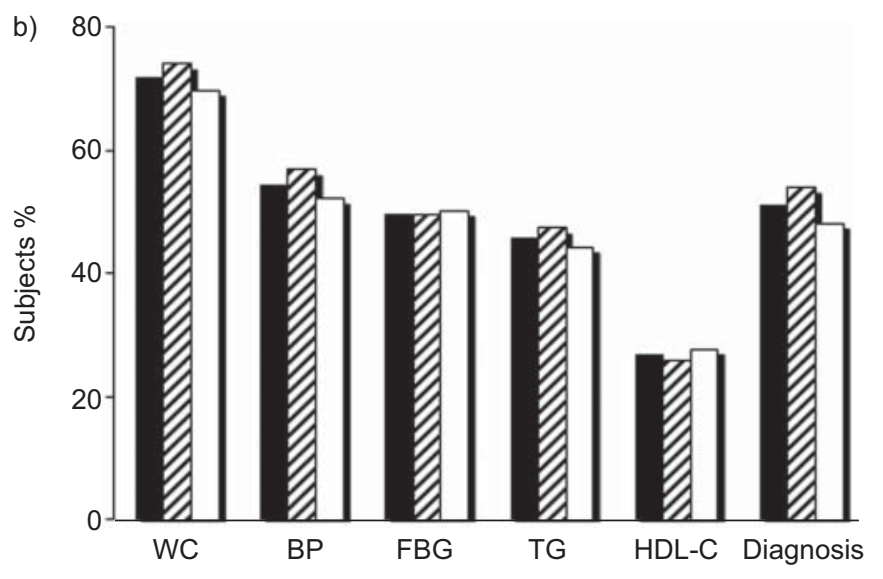

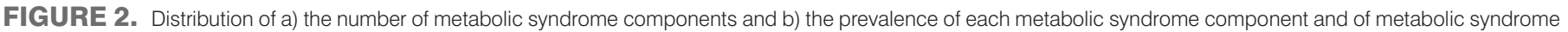

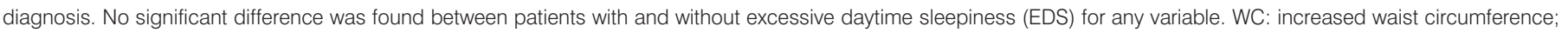
BP: elevated blood pressure; FBG: elevated fasting blood glucose; TG: elevated triglycerides; HDL-C: decreased high-density lipoprotein cholesterol. 


\begin{tabular}{|c|c|c|c|c|c|c|c|c|c|c|c|c|}
\hline \multirow{3}{*}{ TABLE 3} & \multicolumn{6}{|c|}{ AHI } & \multicolumn{6}{|c|}{ Mean $\mathrm{Sa}, \mathrm{O}_{2}$} \\
\hline & \multicolumn{2}{|c|}{ Unadjusted } & \multicolumn{2}{|c|}{ Model $1^{*}$} & \multicolumn{2}{|c|}{ Model $2 \pi$} & \multicolumn{2}{|c|}{ Unadjusted } & \multicolumn{2}{|c|}{ Model $1^{\#}$} & \multicolumn{2}{|c|}{ Model $2^{\pi}$} \\
\hline & $\boldsymbol{\beta}$ & $\mathrm{p}$-value & $\boldsymbol{\beta}$ & $\mathrm{p}$-value & $\beta$ & $\mathrm{p}$-value & $\beta$ & $p$-value & $\beta$ & $\mathrm{p}$-value & $\boldsymbol{\beta}$ & p-value \\
\hline Systolic blood pressure & 0.627 & $<0.001$ & 0.414 & $<0.001$ & 0.395 & 0.001 & -0.056 & $<0.001$ & -0.020 & 0.03 & -0.016 & NS \\
\hline Diastolic blood pressure & 0.752 & $<0.001$ & 0.524 & $<0.001$ & 0.495 & 0.001 & -0.069 & $<0.001$ & -0.039 & $<0.001$ & -0.033 & 0.006 \\
\hline Fasting blood glucose & 0.214 & $<0.001$ & 0.013 & NS & 0.012 & NS & -0.036 & $<0.001$ & -0.009 & NS & -0.009 & NS \\
\hline Serum triglycerides $^{+}$ & 10.401 & $<0.001$ & 1.517 & NS & 0.575 & NS & -1.952 & $<0.001$ & -0.599 & 0.04 & -0.442 & NS \\
\hline HDL cholesterol & -0.161 & 0.02 & -0.010 & NS & 0.037 & NS & 0.037 & $<0.001$ & 0.018 & 0.03 & 0.011 & NS \\
\hline
\end{tabular}

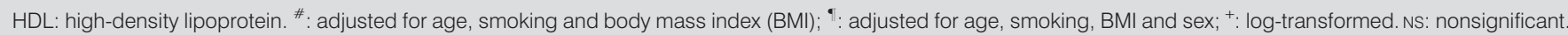

Factors associated with EDS in the entire sample were also analysed. Age (OR 0.979, 95\% CI 0.963-0.995; $\mathrm{p}=0.01$ ) and mean nocturnal $\mathrm{Sa}_{1} \mathrm{O}_{2}$ (OR $0.917,95 \%$ CI $0.869-0.968 ; \mathrm{p}=0.002$ ) were negatively associated with EDS, and explained $20 \%$ of EDS variability.

\section{DISCUSSION}

In a large "real-life" sample of OSA patients at diagnosis, the metabolic syndrome according to NCEP-ATP III criteria occurred in about half of the subjects, and severe OSA was significantly associated with a diagnosis of metabolic syndrome, i.e. occurrence of three or more components of the syndrome. An increase in the number of metabolic syndrome components was associated with worse insulin resistance; correlations between markers of OSA severity and the HOMA index became nonsignificant after adjusting for BMI, indicating a major role of obesity in the relationship between OSA and insulin resistance. Each of the metabolic syndrome components showed crude linear

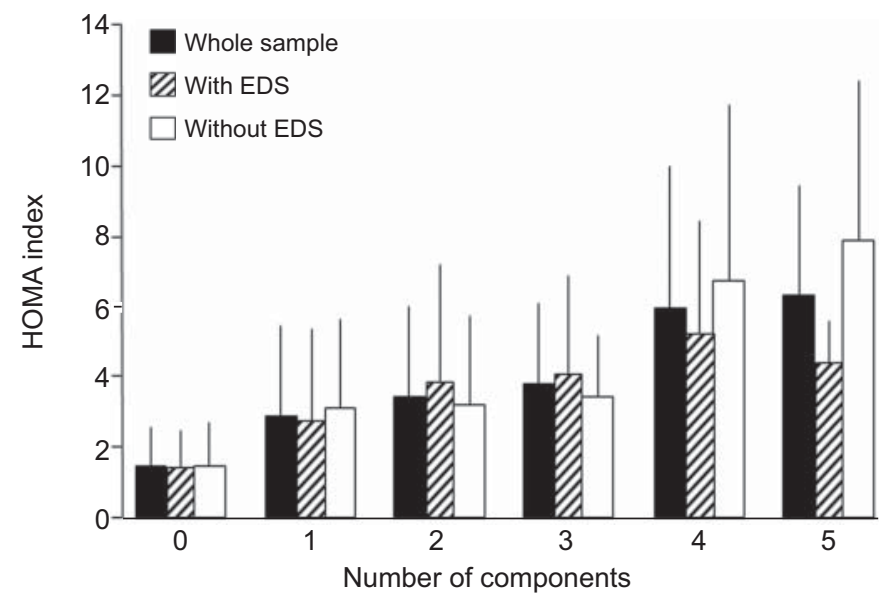

FIGURE 3. Insulin resistance, assessed as Homeostatic Model Assessment (HOMA) index in 288 patients, linearly increased with the number of metabolic syndrome components (Spearman's rho 0.455; $p<0.001$ ). No significant differences were found between patients with and without excessive daytime sleepiness (EDS). The number of subjects in each group can be found in table 4 relationships with markers of OSA severity but, after adjustment for confounders, AHI remained significantly correlated only to blood pressure, and mean $\mathrm{Sa}_{2} \mathrm{O}_{2}$ remained correlated only to waist circumference and diastolic blood pressure. Therefore, the metabolic syndrome was associated with both obesity- and OSA-related variables. EDS was shown to be a poor clinical marker of metabolic abnormalities, and nocturnal intermittent hypoxia and age explained a small fraction of EDS variability.

\section{Prevalence of the metabolic syndrome}

Clinical studies in OSA patients have reported variable prevalence of the metabolic syndrome according to NCEP-ATP III criteria, with values ranging between $23 \%$ and $87 \%$ [14-19]. The largest published series (819 Japanese patients) found prevalence of the metabolic syndrome to be $49.5 \%$ in males and $32 \%$ in females [15]. Our results are similar for male patients, but the prevalence rate of metabolic syndrome in our female patients was higher, possibly due to more severe obesity in our sample.

Case-control studies have reported variable prevalence rates of the metabolic syndrome, or of insulin resistance and metabolic syndrome components [7, 28]. Increased waist circumference, hypertension and increased fasting blood glucose were the

\section{TABLE 4 Subjects assessed by the Homeostatic Model Assessment (HOMA) index (see fig. 3)}

\begin{tabular}{lccc}
$\begin{array}{l}\text { Number of } \\
\text { components }\end{array}$ & Whole sample & With EDS & Without EDS \\
\hline $\mathbf{0}$ & 43 & 24 & 19 \\
$\mathbf{1}$ & 51 & 24 & 27 \\
$\mathbf{2}$ & 80 & 40 & 40 \\
$\mathbf{3}$ & 72 & 43 & 29 \\
$\mathbf{4}$ & 34 & 20 & 14 \\
$\mathbf{5}$ & 8 & 4 & 4 \\
\hline
\end{tabular}

Data are presented as $n$. Subjects were grouped according to the number of metabolic syndrome components and assessment of excessive daytime sleepiness (EDS) 


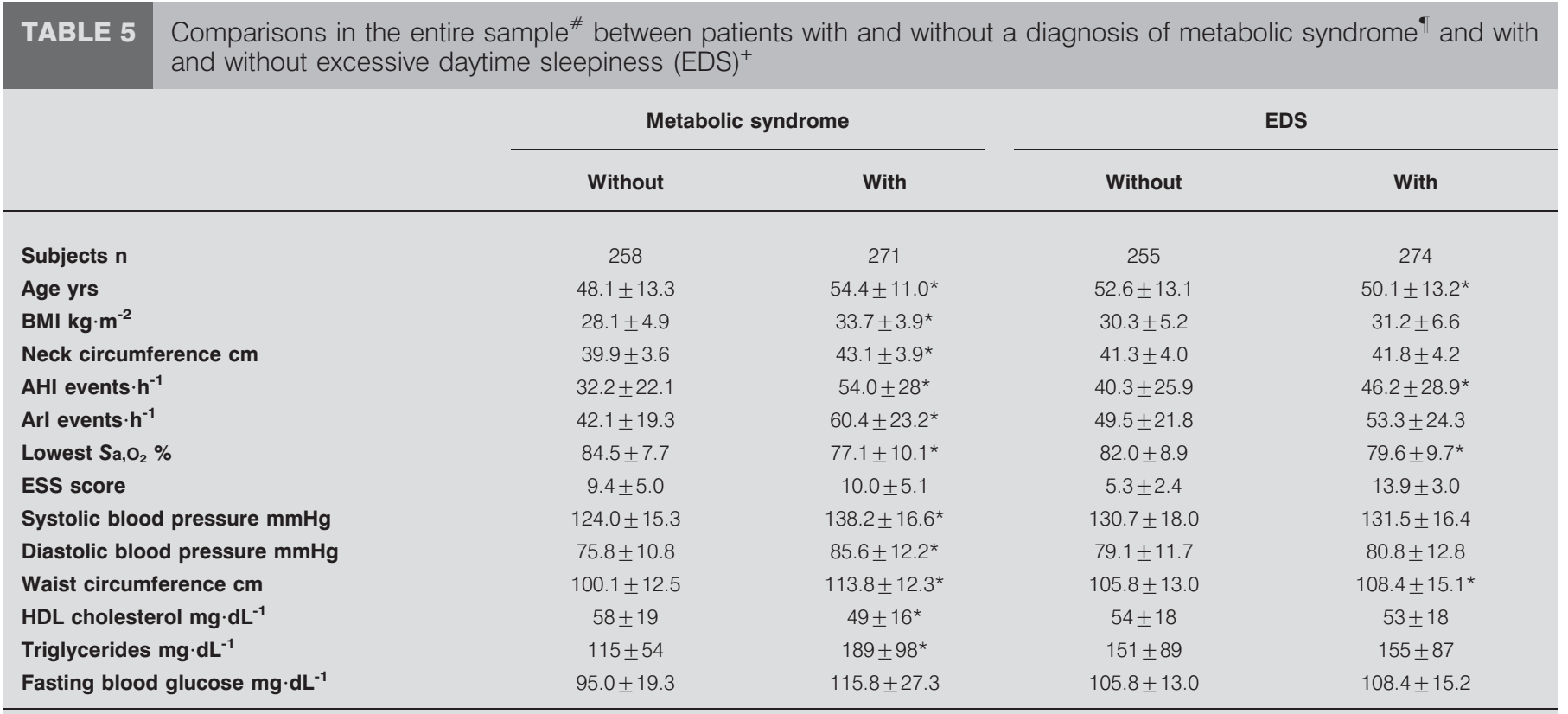

Data are presented as mean \pm SD, unless otherwise stated. BMI: body mass index; $\mathrm{AHI}$ : apnoea/hypopnoea index; Arl: arousal index; $\mathrm{Sa}, \mathrm{O}_{2}$ : arterial oxygen saturation; ESS: Epworth Sleepiness Scale; HDL: high-density lipoprotein. ${ }^{*}: n=529 ;{ }^{\bullet}$ : three or more components; ${ }^{+}$: ESS $\geqslant 10$. *: significant difference for with versus without $(p<0.05)$.

commonest metabolic syndrome components in the present study; these findings agree with those reported by KONO et al. [28]. Conversely, the study by SASANABE et al. [15] reported dyslipidaemia as the third most frequent finding. Finally, population-based studies yielded a prevalence of metabolic syndrome in OSA of between $26 \%$ and 35\% [29-31]. A recent population study reported a $44 \%$ prevalence of metabolic syndrome in females with AHI $>15$ events $\cdot h^{-1}$ [32].

\section{Components of the metabolic syndrome and insulin resistance}

The metabolic syndrome is considered to be the clinical manifestation of insulin resistance [10, 13]. Two studies, however, reported that insulin resistance may not be associated with metabolic syndrome in OSA patients. In the Turkish population, OSA in males was associated with metabolic syndrome but not with insulin resistance [20]. A case-control study reported similar results [7]. The linear increase of the HOMA index with an increasing number of metabolic syndrome components, as found in our study, suggests that this number, known as the metabolic index, may be a clinically useful indicator of the metabolic load in OSA patients. When the data were stratified for OSA severity, occurrence of one or two components of the metabolic syndrome showed similar frequencies in patients with mild, moderate or severe OSA, whereas a diagnosis of metabolic syndrome (i.e. three or more components) was more frequent in severe OSA, and absence of any metabolic syndrome component prevailed in mild OSA. These findings agree with those reported by THEORELLHAGLÖW et al. [32] in a population-based study on females, indicating that the association between OSA and metabolic syndrome is especially strong in patients with severe OSA.

The number of metabolic syndrome components carries prognostic implications. Both all-cause and cardiovascular mortality increased with the number of metabolic syndrome components [11], hypertension being the most potent factor, followed by central obesity and hypertriglyceridaemia. Other studies found increased risk only in patients with three or more metabolic syndrome components [33], or reported that a diagnosis of metabolic syndrome was not superior to the sum of individual risk factors in predicting cardiovascular mortality [34], severity of vascular lesions or progression of atherosclerosis [35]. No longitudinal data are yet available on the prognostic significance of metabolic syndrome components in OSA patients.

Approximately $10 \%$ of our patients did not show any metabolic syndrome component. This subset differed from the rest of the sample, as the subjects were younger, non-obese and had mild OSA. It is possible that absence of metabolic defects represents an early stage in the natural history of OSA, but longitudinal studies are necessary to test this hypothesis. Alternatively, these patients may represent a distinct, still incompletely characterised, clinical OSA phenotype. Interestingly, the metabolic effects of intermittent hypoxia were recently shown to be quite small in lean mice [36]. If the same results were to be shown in humans with OSA, the patients without any metabolic syndrome component may represent a low-risk subgroup, with obvious consequences regarding treatment. Some studies, however, found metabolic abnormalities in non-obese OSA patients, although a diagnosis of metabolic syndrome was not fulfilled [19, 28, 37]. Conversely, both morbidly obese patients [38] and patients with the metabolic syndrome [26] showed worse metabolic variables associated with severe OSA compared with subjects without OSA.

\section{Relationships between metabolic syndrome, OSA and obesity}

Our study found that age, lowest $\mathrm{Sa}_{2} \mathrm{O}_{2}$, BMI, neck circumference and ArI were significantly associated with metabolic 
syndrome by multiple regression analysis, suggesting an independent role of OSA in addition to obesity. Neck circumference was found to independently predict cardiovascular risk in a large population-based study [39], but prevalence of OSA was not assessed, indicating the need to further study the impact of fat distribution on the complex relationship between OSA, obesity and metabolism. Sex may also play a role, as suggested by the results obtained by separate analysis of males and females. In males, markers of central obesity and intermittent hypoxia were significantly associated with metabolic syndrome, whereas in females only age and ArI were significant factors, possibly suggesting a major role of sleep fragmentation in females.

\section{Sleepiness and metabolic syndrome}

EDS has been proposed as a marker of OSA severity, especially for cardiovascular and metabolic outcomes [21-25]. Two casecontrol studies reported that EDS in OSA was associated with insulin resistance, suggesting that it could be used clinically as a marker of cardiometabolic abnormalities. Other studies, however, did not confirm such findings [26, 40]. In our study, EDS correlated negatively with age and mean nocturnal $\mathrm{Sa}_{2} \mathrm{O}_{2}$, and did not affect the relationship between OSA and metabolic variables. The discrepancy between the results of case-control studies and our "real-life" study probably stems from the characteristics of the samples, since highly selected patients without comorbidities were examined in the studies by BARCELÓ et al. [24] and NENA et al. [25]. We acknowledge that the ESS does not assess sleepiness objectively, and lack of multiple sleep latency test data is a major limitation of our study.

\section{Conclusions}

The metabolic syndrome according to NCEP-ATP III criteria occurs in about half of OSA patients at diagnosis, an additional $38.2 \%$ of patients showing one or two metabolic syndrome components. The number of metabolic syndrome components correlated with the HOMA index in OSA patients, and can be used as a clinical marker of insulin resistance. EDS, however, did not turn out to be a sensitive clinical marker of a detrimental metabolic profile in real-life OSA patients.

\section{SUPPORT STATEMENT}

This study was supported by Fondo de Investigaciones Sanitarias (PI070585-PI070598; Instituto Carlos III, Madrid, Spain).

\section{STATEMENT OF INTEREST}

None declared.

\section{ACKNOWLEDGEMENTS}

The authors wish to thank M. Arbones (Pneumology Dept, Hospital Arnau Vilanova, Lleida, Spain) and M. Iglesias (Pneumology Dept, Hospital Universitari Son Dureta, Palma de Mallorca, Spain) for their advice and contributions.

\section{REFERENCES}

1 McArdle N, Hillman D, Beilin L, et al. Metabolic risk factors for vascular disease in obstructive sleep apnea: a matched controlled study. Am J Respir Crit Care Med 2007; 175: 190-195.

2 Marin JM, Carrizo SJ, Vicente E, et al. Long-term cardiovascular outcomes in men with obstructive sleep apnoea-hypopnoea with or without treatment with continuous positive airway pressure: an observational study. Lancet 2005; 365: 1046-1053.

3 Doherty LS, Kiely JL, Swan V, et al. Long-term effects of nasal continuous positive airway pressure therapy on cardiovascular outcomes in sleep apnea syndrome. Chest 2005; 127: 2076-2084.

4 Lévy P, Bonsignore MR, Eckel J. Sleep, sleep-disordered breathing and metabolic consequences. Eur Respir J 2009; 34: 243-260.

5 Louis M, Punjabi NM. Effects of acute intermittent hypoxia on glucose metabolism in awake healthy volunteers. J Appl Physiol 2009; 106: 1538-1544.

6 Barceló A, Barbé F, Llompart E, et al. Effects of obesity on Creactive protein level and metabolic disturbances in male patients with obstructive sleep apnea. Am J Med 2004; 117: 118-121.

7 Gruber A, Horwood F, Sithole J, et al. Obstructive sleep apnea is independently associated with the metabolic syndrome but not insulin resistance state. Cardiovasc Diabetol 2006; 5: 22-29.

8 Sharma SK, Kumpawat S, Goel A, et al. Obesity, and not obstructive sleep apnea, is responsible for metabolic abnormalities in a cohort with sleep-disordered breathing. Sleep Med 2007; 8: 12-17.

9 Otake K, Sasanabe R, Hasegawa R, et al. Glucose intolerance in Japanese patients with obstructive sleep apnea. Intern Med 2009; 48: 1863-1868.

10 Lorenzo C, Okoloise M, Williams K, et al. The metabolic syndrome as predictor of type 2 diabetes: the San Antonio Heart Study. Diabetes Care 2003; 26: 3153-3159.

11 Ho JS, Cannaday JJ, Barlow CE, et al. Relation of the number of metabolic syndrome risk factors with all-cause and cardiovascular mortality. Am J Cardiol 2008; 102: 689-692.

12 Simmons RK, Alberti KG, Gale EA, et al. The metabolic syndrome: useful concept or clinical tool? Report of a WHO Expert Consultation. Diabetologia 2010; 53: 600-605.

13 Alberti KG, Eckel RH, Grundy SM, et al. Harmonizing the metabolic syndrome: a joint interim statement of the International Diabetes Federation Task Force on Epidemiology and Prevention; National Heart, Lung, and Blood Institute; American Heart Association; World Heart Federation; International Atherosclerosis Society; and International Association for the Study of Obesity. Circulation 2009; 120: $1640-1645$.

14 Coughlin SR, Mawdsley L, Mugarza JA, et al. Obstructive sleep apnoea is independently associated with an increased prevalence of metabolic syndrome. Eur Heart J 2004; 25: 735-741.

15 Sasanabe R, Banno K, Otake K, et al. Metabolic syndrome in Japanese patients with obstructive sleep apnea syndrome. Hypertens Res 2006; 29: 315-322.

16 Peled N, Kassirer M, Shitrit D, et al. The association of OSA with insulin resistance, inflammation and metabolic syndrome. Respir Med 2007; 101: 1696-1701.

17 Parish JM, Adam T, Facchiano L. Relationship of metabolic syndrome and obstructive sleep apnea. J Clin Sleep Med 2007; 3: $467-472$.

18 Agrawal S, Sharma SK, Sreenivas V, et al. Stepped approach for prediction of syndrome $Z$ in patients attending sleep clinic: a north Indian hospital-based study. Sleep Breath 2011; [Epub ahead of print DOI: $10.1007 / \mathrm{s} 11325-011-0548-3]$.

19 Akahoshi T, Uematsu A, Akashiba T, et al. Obstructive sleep apnoea is associated with risk factors comprising the metabolic syndrome. Respirology 2010; 15: 1122-1126.

20 Onat A, Hergenç G, Uyarel H, et al. Obstructive sleep apnea syndrome is associated with metabolic syndrome rather than insulin resistance. Sleep Breath 2007; 11: 23-30.

21 Kapur VK, Resnick HE, Gottlieb DJ, et al. Sleep disordered breathing and hypertension: does self-reported sleepiness modify the association? Sleep 2008; 31: 1127-1132.

22 Lombardi C, Parati G, Cortelli P, et al. Daytime sleepiness and neural cardiac modulation in sleep-related breathing disorders. J Sleep Res 2008; 17: 263-270. 
23 Ronksley PE, Hemmelgarn BR, Heitman SJ, et al. Obstructive sleep apnoea is associated with diabetes in sleepy subjects. Thorax 2009; 64: 834-839.

24 Barceló A, Barbé F, de la Peña $\mathrm{M}$, et al. Insulin resistance and daytime sleepiness in patients with sleep apnoea. Thorax 2008; 63: 946-950.

25 Nena E, Steiropoulos P, Papanas N, et al. Sleepiness as a marker of glucose deregulation in obstructive sleep apnea. Sleep Breath 2011; [Epub ahead of print DOI: 10.1007/s11325-010-0472-y].

26 Drager LF, Lopes HF, Maki-Nunes C, et al. The impact of obstructive sleep apnea on metabolic and inflammatory markers in consecutive patients with metabolic syndrome. PLoS One 2010; 5: e12065.

27 Matthews DR, Hosker JP, Rudenski AS, et al. Homeostasis model assessment: insulin resistance and beta-cell function from fasting plasma glucose and insulin concentrations in man. Diabetologia 1985; 28: 412-419.

28 Kono M, Tatsumi K, Saibara T, et al. Obstructive sleep apnea syndrome is associated with some components of metabolic syndrome. Chest 2007; 131: 1387-1392.

29 Lam JC, Lam B, Lam CL, et al. Obstructive sleep apnea and the metabolic syndrome in community-based Chinese adults in Hong Kong. Respir Med 2006; 100: 980-987.

30 Chin K, Oga $\mathrm{T}$, Takahashi K, et al. Associations between obstructive sleep apnea, metabolic syndrome, and sleep duration, as measured with an actigraph, in an urban male working population in Japan. Sleep 2010; 33: 89-95.

31 Nieto FJ, Peppard PE, Young TB. Sleep disordered breathing and metabolic syndrome. Wisc Med J 2009; 108: 263-265.
32 Theorell-Haglöw J, Berne C, Janson C, et al. The role of obstructive sleep apnea in metabolic syndrome: a population-based study in women. Sleep Med 2011; 12: 329-334.

33 Sundström J, Vallhagen E, Risérus U, et al. Risk associated with the metabolic syndrome versus the sum of its individual components. Diabetes Care 2006; 29: 1673-1674.

34 Baldassarre D, Werba JP, Castelnuovo S, et al. The metabolic syndrome predicts carotid intima-media thickness no better than the sum of individual risk factors in a lipid clinic population. Atherosclerosis 2010; 210: 214-219.

35 Bayturan O, Tuzcu EM, Lavoie A, et al. The metabolic syndrome, its component risk factors, and progression of coronary atherosclerosis. Arch Intern Med 2010; 170: 478-484.

36 Drager LF, Li J, Reinke C, et al. Intermittent hypoxia exacerbates metabolic effects of diet-induced obesity. Obesity (Silver Spring) 2011; 19: 2167-2174.

37 Lin QC, Zhang XB, Chen GP, et al. Obstructive sleep apnea is associated with some components of metabolic syndrome in nonobese adults. Sleep Breath 2011; [Epub ahead of print DOI: 10.1007/s11325-011-0544-7].

38 Gasa M, Salord N, Fortuna AM, et al. Obstructive sleep apnoea and metabolic impairment in severe obesity. Eur Respir J 2011; 38: 1089-1097.

39 Preis SR, Massaro JM, Hoffmann U, et al. Neck circumference as a novel measure of cardiometabolic risk: the Framingham Heart study. J Clin Endocrinol Metab 2010; 95: 3701-3710.

40 Drager LF, Bortolotto LA, Maki-Nunes C, et al. The incremental role of obstructive sleep apnoea on markers of atherosclerosis in patients with metabolic syndrome. Atherosclerosis 2010; 208: 490-495. 